\title{
Enhancing Underexposed Images Preserving the Original Mood
}

\author{
Silvia Corchs and Francesca Gasparini \\ DISCo (Dipartimento di Informatica, Sistemistica e Comunicazione), \\ University of Milano-Bicocca, Viale Sarca 336, 20126 Milano, Italy \\ \{corchs, gasparini\}@disco.unimib.it
}

\begin{abstract}
In the present article we focus on enhancing the contrast of images with low illumination that present large underexposed regions. Most of these images represent night images. When applying standard contrast enhancement techniques, usually the night mood is modified, and also a noise over-enhancement within the darker regions is introduced. In a previous work we have described our local contrast correction algorithm designed to enhance images where both underexposed and overexposed regions are simoultaneously present. Here we show how this algorithm is able to automatically enhance night images, preserving the original mood. To further improve the performance of our method we also propose here a denoising procedure where the strength of the smoothing is a function of an estimated level of noise and it is further weighted by a saliency map. The method has been applied to a proper database of outdoor and indoor underexposed images. Our results have been qualitatively compared with well know contrast correction methods.
\end{abstract}

Keywords: local contrast enhancement, underexposed images, night images.

\section{Introduction}

Global and local contrast correction algorithms have become very popular to improve the quality of the captured images (like those obtained with mobile devices among others) when underexposed and overexposed regions are simultaneously present within the image. In the literature, many algorithms have been proposed, from histogram equalization-type techniques [17], [2] to other types of methods like the Retinex model 6] or local contrast corrections methods, where non linear masking is used in order to perform the local processing [13], 16].

In the present article we focus on enhancing the contrast of images with low illumination that present important or large underexposed regions. Most of these images depict night scenes typically acquired outdoor, or indoor scenes acquired with very low level of illumination (for instance inside pubs and discoteques, during parties, etc.). In these particular cases, when applying standard contrast enhancement techniques, usually the original mood of the image changes, and also a noise over-enhancement within the darker regions can be introduced. For 

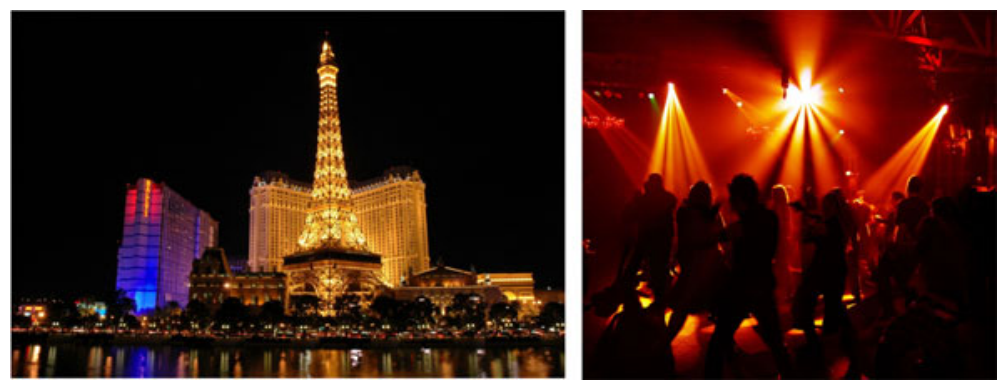

Fig. 1. Example of underexposed images. Left, an outdoor night image. Right, an indoor image.

images taken with digital cameras, darker regions will contain more noise than the brighter ones. In Figure 1 an example of a night image and an indoor underexposed image are shown.

A contrast correction strategy applied to these images should find a trade-off between enhancing the visibility of details and preserving the original mood of the image. Moreover, it is also desirable to control the noise level in the output image.

In a previous work, [16, we have described our local contrast correction algorithm designed to enhance images where both underexposed and overexposed regions are simoultaneously present. In this paper we show how our algorithm is able to enhance this kind of strongly underexposed images, preserving the original mood. We add to our method an automatic white balance algorithm [3], that is able to mimic the behaviour of our visual system discounting cast of low and middle intensity, while mantaining strong cast due to a particular light condition, such as in the case of images acquired in a discoteque with coloured lights (see for example the image on the right of Figure 1). To further improve the performance of our method, we also propose here a denoising procedure. The denoising problem has been widely addressed within the literature 4, 14, 15, 19.

Noise estimation is a difficult task and different approaches have been proposed [8] [18, 11] [1. While it is often assumed that noise is additive and Gaussian, for the particular case of underexposed images we note that it is strongly dependent on the image intensity. Moreover, the noise in digital images is both chromatic and achromatic.

Thus in our method we propose to move to the YCbCr color space and estimate the noise level for each channel and apply different denoising strategies for each of them.

For the intensity channel Y, we propose to apply a modified version of the bilateral filter introduced by Tomasi and Manduchi, [19. In order to obtain a selective denoise, the smoothing effect of the bilateral filter is weighted by a saliency map of the image. Saliency is a concept which states that there are regions in a scene that are more relevant than their neighbors and hence draw attention. Based on a biologically plausible architecture, several authors implemented a 
saliency map model mainly using color, intensity and orientation cues to predict salient regions, [912] The idea of performing different enhancing strategies with respect to the salience of the regions of an image where previously used by many authors, see for example Gasparini et al [7. As the high frequencies are more visible in the intensity channel with respect to the chromatic ones, we apply for the latter ones the well known Wiener filter, instead of the bilateral filter.

The paper is organized as follows. In section 2 our local contrast correction algorithm is summarized, underlying its behaviour with respect to the images considered in this work. In section 3 the automatic white balance algorithm here applied is briefly described and some example images are reported. The concept of saliency and the saliency map here adopted are described in section 4, while in section 5 the denoise module here proposed, function of the estimated level of noise and weighted by a saliency map, is described. Finally in section [6 we present the experimental results, showing some images processed by our whole procedure and comparing qualitatively our outputs with well known methods such as Retinex and the local exponential correction introduced by Moroney. Section 7 summarizes the conclusions.

\section{Local Contrast Correction Algorithm}

The Local Contrast Correction (LCC) algorithm here adopted is based on a local and image dependent exponential correction [16]. This algorithm aims to correct images that simultaneously present overexposed and underexposed regions. The LCC algorithm is developed starting from Moroney's technique [13, where the exponent of a gamma correction like function is not a constant but depends on the point to be corrected, its neighbouring pixels and on the global characteristic of the image. The corrected image $I_{c}(i, j)$ is obtained as follows:

$$
I_{c}(i, j)=I(i, j)^{\alpha} \frac{\left(\frac{128-m a s k(i, j)}{128}\right)}{}
$$

where $\alpha$ is a parameter depending on the image properties. For low contrast images, where a stronger correction is needed, $\alpha$ should be high, while for better contrasted images, $\alpha$ should diminish towards 1, which corresponds to no correction (See Schettini et al. [16] for more details). In equation 1, mask $(x, y)$ is an inverted low-pass version of the intensity of the input image. In the LCC method, instead of the Gaussian filter of the Moroney proposal, the bilateral filter of Tomasi and Manduchi is used [19]. Bilateral filtering smoothes images while preserving edges, by means of a nonlinear combination of nearby image values. The bilateral filter combines grey levels or colors based on both their geometric closeness and their photometric similarity, and prefers near values to distant values in both spatial and intensity domains. The idea developed by the bilateral filter is to combine domain and range Gaussian filtering (depending respectively on a spatial standard deviation $\sigma_{s}$ and on a range standard deviation $\left.\sigma_{r}\right)$. In this way, both geometric and photometric locality are simultaneously enforced. The mask corresponding to the bilateral filter approach is defined over a window of size $(2 K+1)(2 K+1)$ and is given by: 

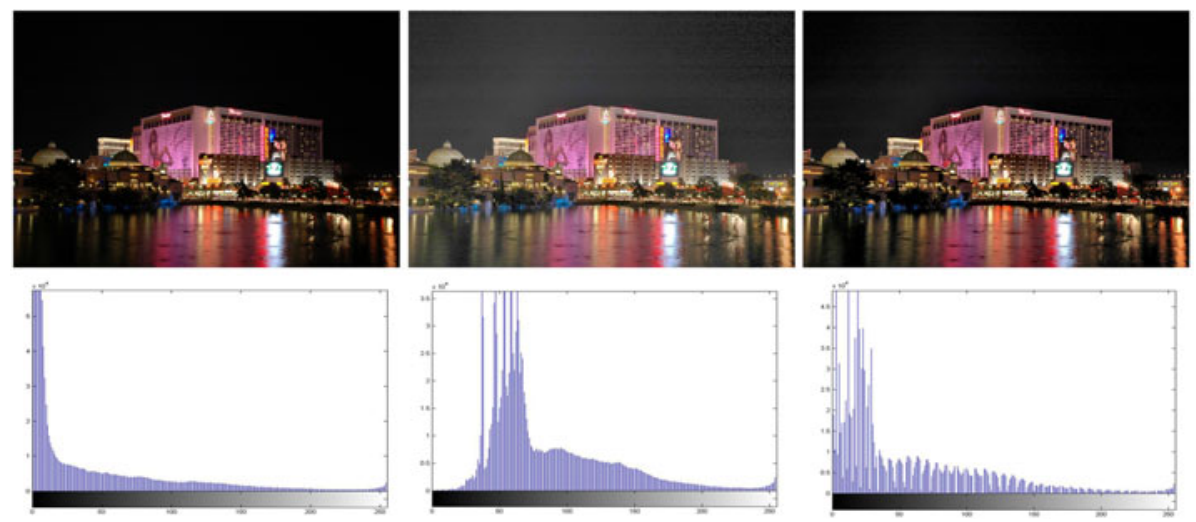

Fig. 2. Original image (left column); the result after applying the local esponential correction of equation 1 (middle column); the final enhanced image, after the stretching and clipping adjustement (right column). Corresponding histograms of the intensity channel are reported below the images.

$$
\begin{aligned}
\operatorname{mask}(i, j)= & \frac{1}{k(i, j)} \sum_{p=i-K}^{i+K} \sum_{q=j-K}^{j+K} \exp \left\{-\frac{1}{2 \sigma_{s}^{2}}\left[(i-p)^{2}+(j-q)^{2}\right]\right\} \\
& \exp \left\{-\frac{1}{2 \sigma_{r}^{2}}\left[I_{i n v}(i, j)-I_{i n v}(p, q)\right]^{2}\right\} I_{i n v}(p, q)
\end{aligned}
$$

where $I_{i n v}(i, j)=255-I(i, j)$ and $k(i, j)$ is a normalization factor:

$$
\begin{aligned}
k(i, j)= & \sum_{p=i-K}^{i+K} \sum_{q=j-K}^{j+K} \exp \left\{-\frac{1}{2 \sigma_{s}^{2}}\left[(i-p)^{2}+(j-q)^{2}\right]\right\} \\
& \exp \left\{-\frac{1}{2 \sigma_{r}^{2}}\left[I_{i n v}(i, j)-I_{\text {inv }}(p, q)\right]\right\}
\end{aligned}
$$

In the present implementation, working with color images, we apply the rule of equation 1 to the intensity component in the $\mathrm{YCbCr}$ color space. From a deeper analysis of the intensity histogram before and after the proposed local correction, it comes that, despite a better occupation of the grey levels, the overall contrast enhancement is not satisfying. In fact, the new histogram is more spread than the original but it is moved and concentrated around the middle values of the range. The effect that makes the processed image greyish is intrinsic in the mathematic formulation of equation 1 adopted for the local correction. To overcome this problem a further step of contrast enhancement, consisting of a stretching and clipping procedure, is added . To determine the strength of the stretching and thus the number of bins to be clipped, it is considered how the darker regions occupy the intensity histogram before and after the LCC algorithm. Pixels belonging to a dark area, such as a dark object, or a dark background (for example the sky during the night) usually occupy a narrow and peaked group of 
bins at the beginning of the intensity histogram. These pixels should populate more or less the same bins after a contrast enhancement algorithm. On the other hand, pixels of regions that create a more spread histogram peak, after the same algorithm must populate an even more spread region of the histogram. In Figure 2 left, an image with a dark background that shuold be preserved after the contrast enhancement procedure is shown; the same image after the esponential correction is depicted in the middle column, while the final image after the stretching step is shown on the right. The corresponding histograms are shown below the images. More details can be found in [16].

\section{Automatic White Balance}

The algorithm proposed uses both the chromaticity and the intensity of the image to estimate the illuminant, and performs the compensation by a diagonal transforms. In particular it combines a spatial segmentation process with empirical designed weighting functions aimed to select the scene objects containing more information for the light chromaticity estimation. The algorithm is a modified
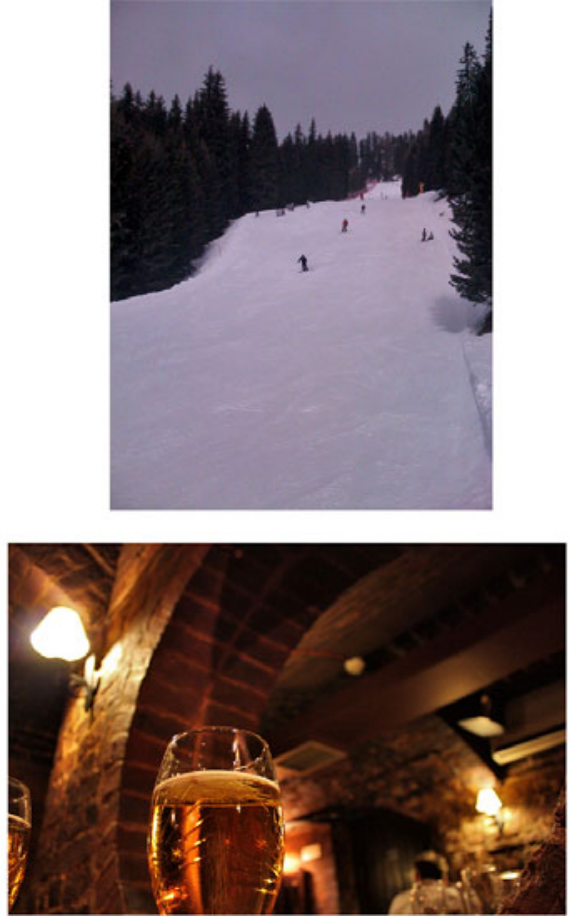
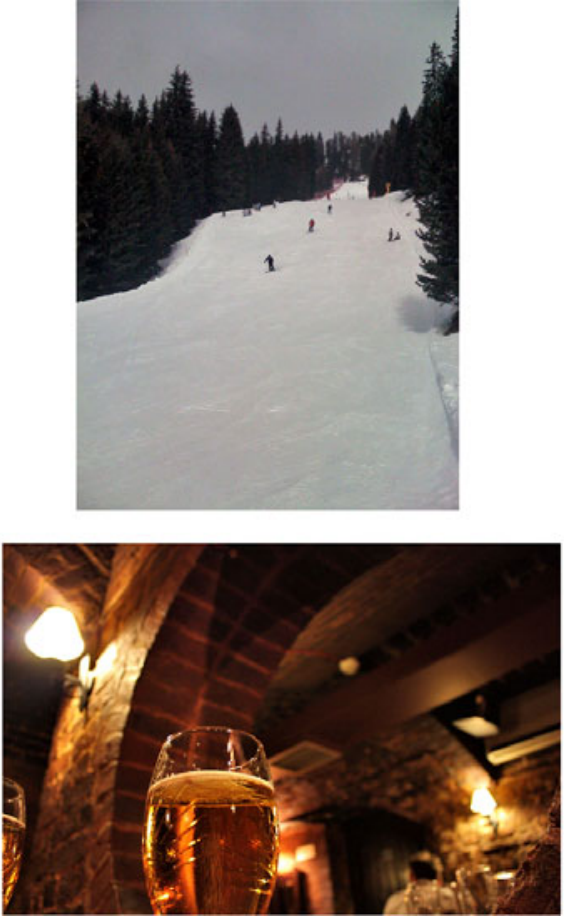

Fig. 3. First row, left) an example of image with a dominant color that should be removed; right) corresponding color corrected output. Second row, left) an image whose dominant color characterizes the mood of the scene and thus should be preserved; right) corresponding output. 
version of the traditional white patch algorithm. The main peculiarity of this color balancing lies in how it determines the region to be set at white, called white balancing region (WB region). Accordingly to wide accepted results that not all the pixels have the same significance in terms of information content of scene illuminant, it takes into account both chromaticity, intensity and spatial information of the image data. In particular it works by combining a spatial segmentation process and proper weighting profiles aimed to select the scene objects containing more information for the light chromaticity estimation. A particular charateristic of this algorithm is that it tries to simulate our visual system, discounting only low or middle intensity casts, that our visual system is ususally able to compensate, leaving unchanged the dominant color that is need to keep the mood of the acquired scene. In Figure 3 one image whose dominant color should be removed, and one image where the dominant color shold remain unchanged are reported in the first and second row respectively. The output of our automatic white balance algorithm is depicted near the corresponding original images. A detailed description of the method can be found in 3 .

\section{Saliency Map}

Saliency is a concept which states that there are regions in a scene that are more relevant than their neighbors and hence draw attention. In the present work we use the contrast-based saliency map proposed by Ma and Zhang [12. The basic algorithm divides the image into small rectangular tiles. At each tile, a contrast

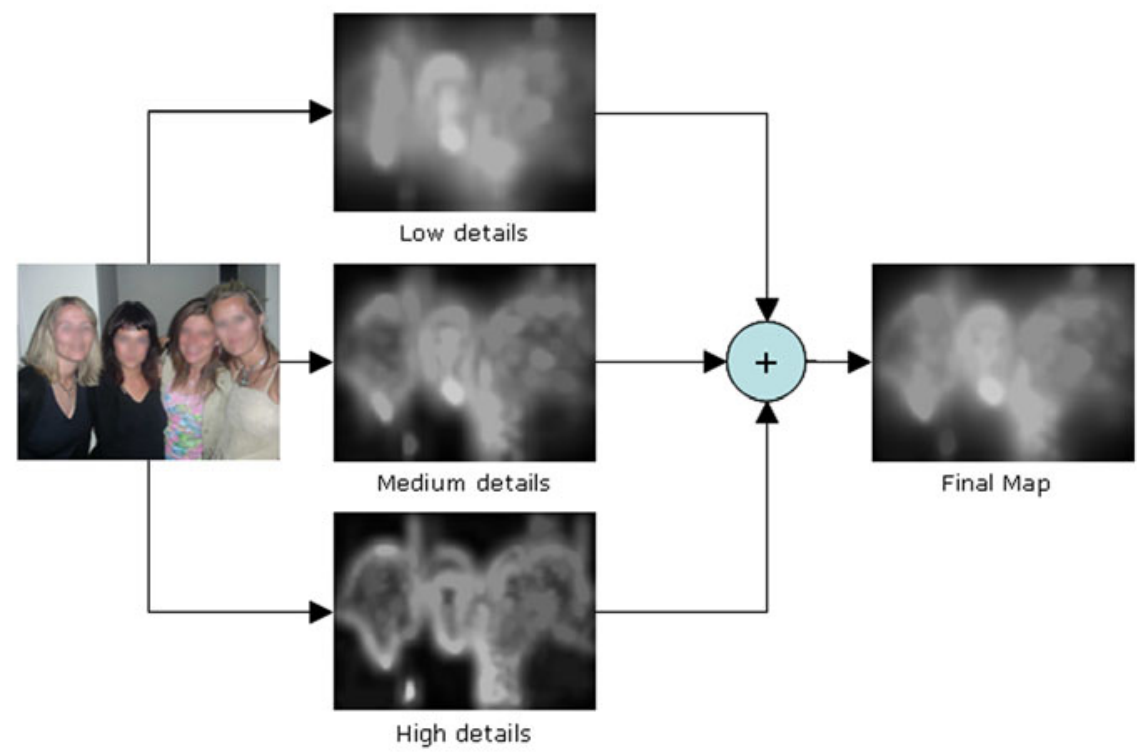

Fig. 4. Original image (left column), the three levels of saliency maps (middle column), the final multi-level saliency map (right column). Courtesy of Ciocca and Schettini [5]. 

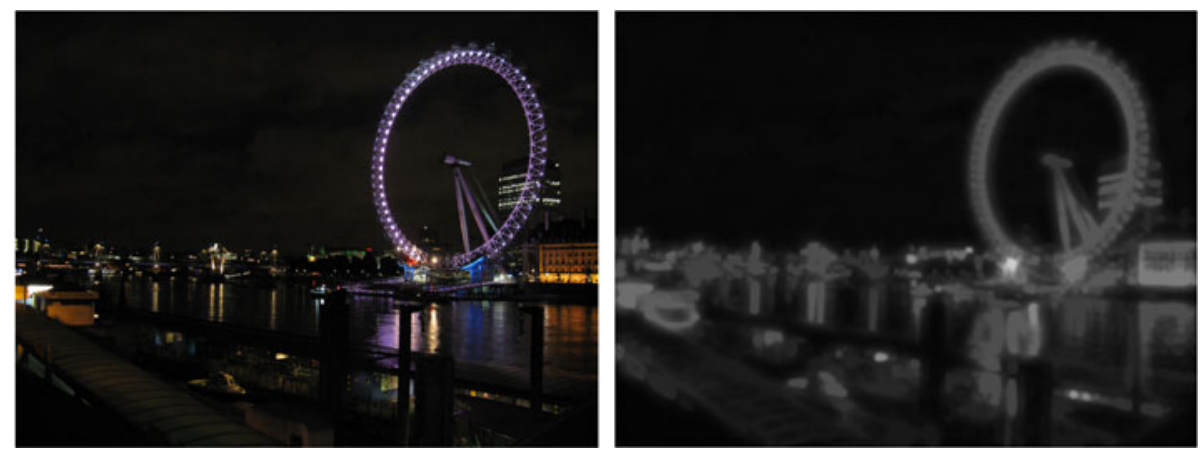

Fig. 5. Example of the saliency map adopted in our method, obtained using only the high details

score is computed from the differences of average colors between the given tile and its neighbor's tiles. The contrast score expresses the saliency of the pixels in the tile. The contrast scores of all the tiles define the saliency map of the image. The size of the tiles, and the size of the neighborhoods determine the dimensions of the salient areas that can be detected. The basic, single scale algorithm has been extended by Ciocca and Schettini [5] to compute three different levels of saliency maps. Using neighborhoods of increasing size, each aimed at a particular level of detail (small, medium and large) a multi-level saliency map was formulated. In Figure 4 the original image, the corresponding three different levels of saliency maps and the final multi-level saliency map are shown.

In the present work we choose to use only the high-details saliency map. In this way for each pixel $(x, y)$ of the contrasted image $I_{c}(x, y)$ the saliency map called $\operatorname{SalMap}(x, y)$, is obtained. As an example, in Figure 5 an image of our database and the corresponding saliency map are shown.

\section{Salience-Adaptive Denoising Module}

For underexposed images, noise is not simply additive, but it is also strongly dependent on the image intensity level. Moreover, noise changes depending on the exposure setting and camera model and it can also vary within an individual image. Therefore, for the images we address in the present article, we ignore what type of noise is present in the image (addditive and/or multiplicative, chromatic and/or achromatic). For this reason, we are not interested here in estimating the noise level as a function of the image intensity. We are mainly interested in estimating noise within the darker regions. In these regions the original Signal to Noise Ratio (SNR) is significantly low and any contrast correction will increase the noise level, further reducing the initial SNR. We select as dark regions those corresponding to the first peak of the intensity histogram of the original image. The noise level will be estimated within these regions in the contrast corrected image, as we are interested in the noise increase due to the enhancement. As the 

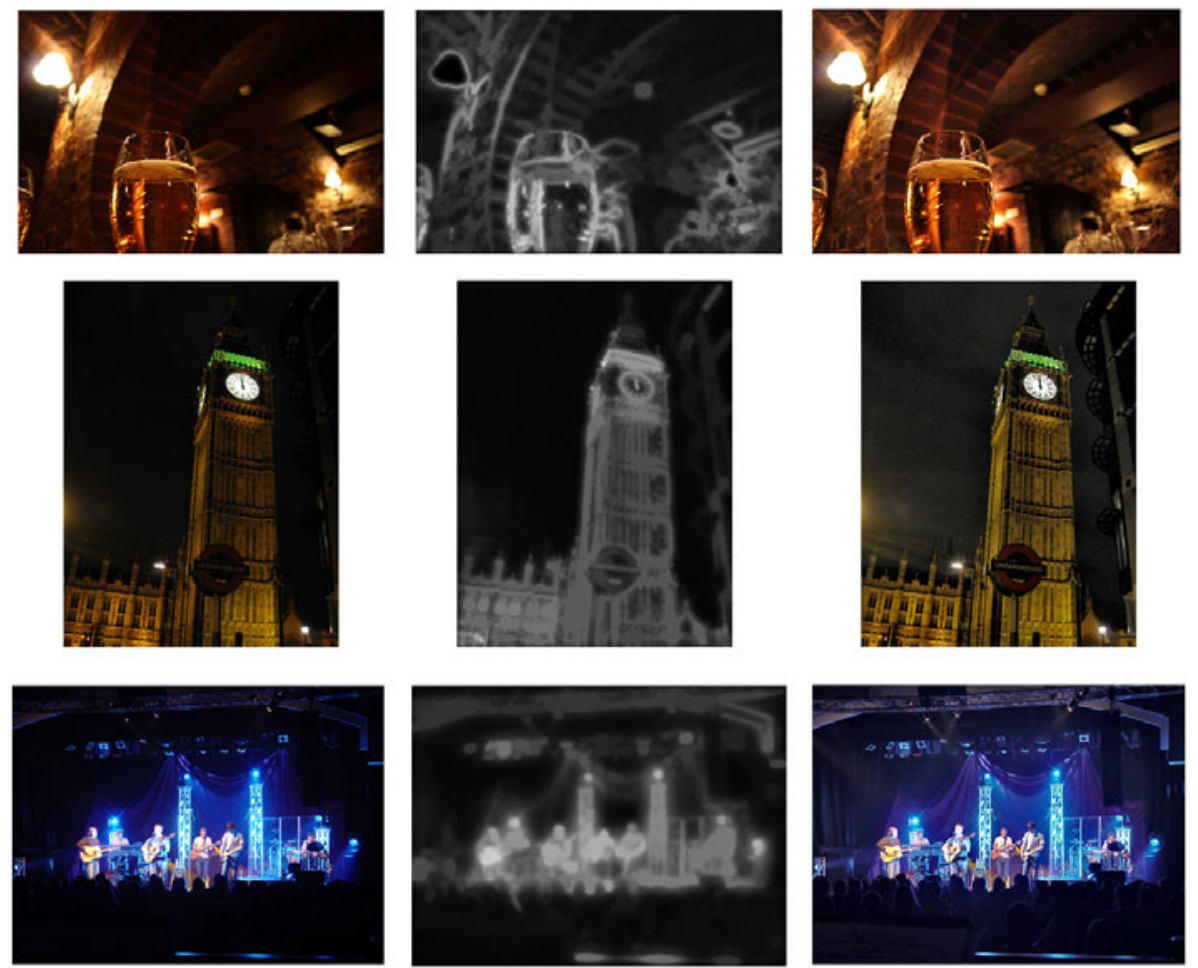

Fig. 6. Left: underexposed original images; middle: corresponding saliency maps; right: outputs of our whole method

noise in digital images is both chromatic and achromatic, we move to the $\mathrm{YCbCr}$ color space and we estimate noise for each channel, evaluating the corresponding standard deviations within the selected regions.

In our denoising module we apply different strategies to intensity and color channels. For the intensity channel $Y$ we adopt a modified version of the bilateral filter introduced by Tomasi and Maduchi [19] and described in section 2, In our algorithm, the standard deviation $\sigma_{r}$ of the Gaussian function in the range domain is related to the estimated intensity noise $\sigma_{\text {noise }}$. In particular, following the proposal of Liu et al [10] we set:

$$
\sigma_{r}=1.95 \sigma_{\text {noise }}
$$

Moreover, the strength of the filtering is weighted by the saliency map $\operatorname{SalMap}(x, y)$ defined above, so that regions more visually salient are filtered less than region less significant. This effect is obtained making $\sigma_{r}$ spatially varying, defining a new $\hat{\sigma}_{r}(x, y)$ as follows:

$$
\hat{\sigma}_{r}(x, y)=\sigma_{r}(1-\operatorname{SalMap}(x, y))
$$


On the other hand, for each of the color channels $\mathrm{Cb}$ and $\mathrm{Cr}$, we apply a Wiener filter, which is a filter adaptive with respect to the level of noise and specifically designed for additive noise. In our algorithm the reference value of noise for each channel is estimated as described above.

\section{Results and Discussion}

We have tested our method on a datasets of more than 40 images acquired with digital cameras of medium/high quality. Thus the images considered have dimension between 4 and 6 Mpixels. For these dimensions we have applied the rule of equation 4 to estimate the $\sigma_{r}$ of the bilateral filter. We have noticed that the factor of this equation should be tuned with respect to the dimension of the processed images. How to tune this parameter will be object of a future work. In Figure [6] the results of the proposed method are shown for some example images. On the left the underexposed original images are shown, in the middle the corresponding saliency maps here adopted are reported, while the output of our whole method can be seen on the right.

In order to compare the results, it is not easy to define reliable no reference quality metrics with normalized range of values. Moreover, it is even more difficult
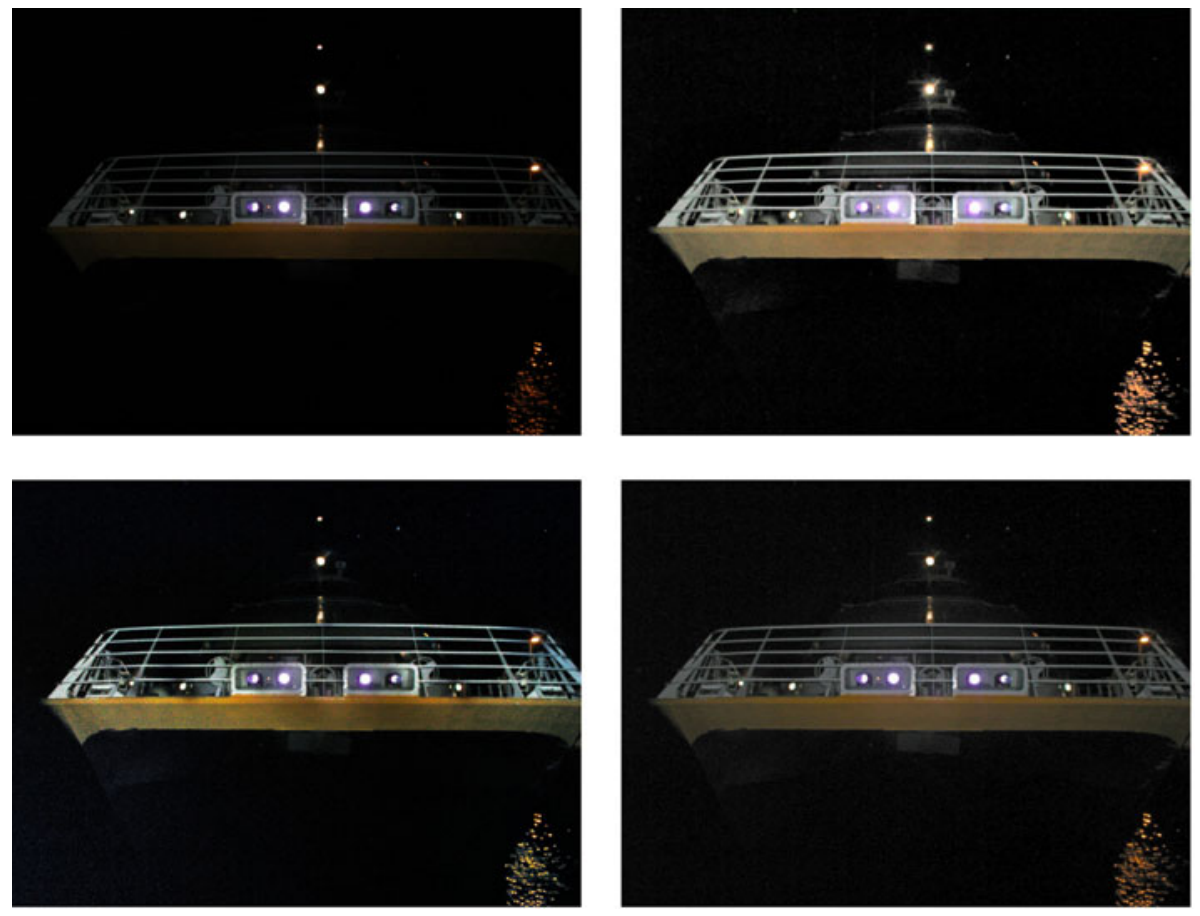

Fig. 7. Original image (top row, left). Image processed with our method (top row, right). Retinex and Moroney results respectively bottom row, left and right. 

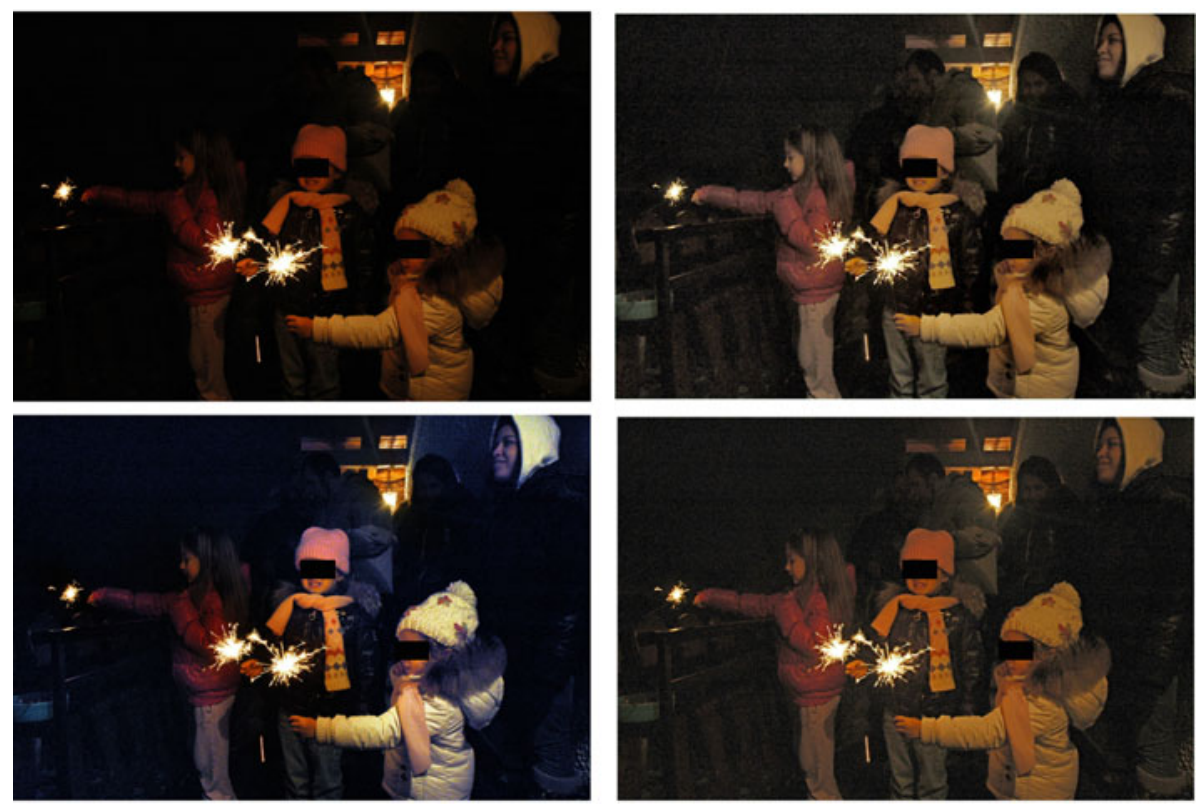

Fig. 8. Original image (top row, left). Image processed with our method (top row, right). Retinex and Moroney results respectively bottom row, left and right.

in the present case where the reduction of noise, the sharpness of the image and the color balancing should be simoultaneously taken into account. Therefore, we only report here qualitatively comparisons with well known methods available in the literature. In particular we have considered the Frankle and McCann version of Retinex [6], as an example of algorithm that not only increases the contrast but also performs a color balancing, and the local exponential correction of Moroney [13. as a typical example of local correction that shows good performance in case of low contrasted images with both under and over-exposed regions but that is not designed to enhanced images with a very low SNR in the darker regions. Two example sets of images are shown in Figures 7 and 8 .

From a qualitative visual analysis we can note that for this kind of images the Retinex method can introduce color distortions, while the Moroney results show grayish appearance. Our method enhnaces the details, preserving the original mood and keeping the noise whitin an acceptable level from a perceptive point of view. A better analysis of the results requires also a subjective psychovisual test, with more images and contents, and involving in the tests a proper number of observers.

\section{Conclusions}

In this work we have presented an adaptive enhancement procedure especially suited for underexposed images, where the noise level of the darker regions usually 
increases significantly after common contrast processing. In particular we were not interested here in enhancing all the details of the underexposed images but instead we were interested in preserving the dark mood typical of night images or of indoor images acquired in particular light contitions (such as those acquired in pubs, discoteques, etc.). To further improve the quality of the processed images, our algorithm adds a proper denoising module after a local and image dependent contrast correction and automatic white balance procedure. This denoising module is 'salience-adaptive' as it is weighted by the saliency map of the image. Moreover, its strength is piloted by the noise level of the image. Experimental results and a visual comparison with well known methods, reveal that our method is able to preserve the original mood of the acquired scenes, keeping the noise level low.

\section{References}

1. Aja-Fernndez, S., Vegas-Snchez-Ferreroa, G., Martn-Fernndez, M., Alberola-Lpez, C.: Automatic noise estimation in images using local statistics. additive and multiplicative cases. Image and Vision Computing 27, 756-770 (2009)

2. Arici, T., Dikbas, S., Altunbasa, Y.: A histogram modification framework and its application for image contrast enhancement. IEEE Transactions on Image Processing 18, 1921-1935 (2009)

3. Bruna, A., Naccari, F., Gasparini, F., Schettini, R.: Multidomain pixel analysis for illuminant estimation. In: Proc. of SPIE Digital Photography II, vol. 6069, pp. 115-122 (2006)

4. Buades, A., Coll, B., Morel, J.M.: A review of image denoising algorithms, with a new one. Simul. 4, 490-530 (2005)

5. Ciocca, C., Schettini, R.: Multiple image thumbnailing. Proceedings of SPIE Digital Photography VI, vol. 7537, p. 75370S (2010)

6. Frankle, J., McCann, J.: Method and apparatus for lightness imaging. US Patent 4, 384, 386 (1983)

7. Gasparini, F., Corchs, S., Schettini, R.: Low quality image enhancement using visual attention. Optical Engineering letters 46, 040502 (2007)

8. Immerkaer, J.: Fast noise variance estimation. Computer Vision and Image Understanding 64, 300-302 (1996)

9. Itti, L., Koch, C.: A saliency-based search mechanism for overt and covert shifts of visual attention. Vision Research 40, 1489-1506 (2000)

10. Liu, C., Freeman, W., Szeliski, R., Kang, S.B.: Noise estimation from a single image. In: IEEE Computer Society Conference on Computer Vision and Pattern Recognition, 2006, vol. 1, pp. 901-908 (2006)

11. Liu, C., Szeliski, R., Kang, S.B., Zitnick, C.L., Freeman, W.T.: Automatic estimation and removal of noise from a single image. IEEE Transactions on Pattern Analysis and Machine Intelligence 30, 299-314 (2008)

12. Ma, Y., Zhang, H.J.: Contrast-based image attention analysis by using fuzzy growing. In: Proc. of the Eleventh ACM International Conference on Multimedia, pp. 374-381 (2003)

13. Moroney, N.: Local colour correction using non-linear masking. In: IS\&T/SID Eighth Color Imaging Conference, pp. 108-111 (2000)

14. Perona, P., Malik, J.: Scale-space and edge detection using anisotropic diffusion. IEEE Trans. on Pattern Analysis and Machine Intelligence 12, 629-639 (1990) 
15. Portilla, J., Strela, V., Wainwright, M.J., Simoncelli, E.P.: Image denoising using scale mixtures of Gaussians in the wavelet domain 12, 1338-1351 (2003)

16. Schettini, R., Gasparini, F., Corchs, S., Marini, F., Capra, A., Castorina, A.: A contrast image correction method. Journal of Electronic Imaging 19, 023005 (2010)

17. Stark, A.: Adaptive image contrast enhancement using generalizations of histogram equalization. IEEE Transactions on Image Processing 9, 889-896 (2000)

18. Tai, S., Yang, S.: A fast method for image noise estimation using laplacian operator and adaptive edge detection. In: Communications, Control and Signal Processing ISCCSP, pp. 1077-1081 (2008)

19. Tomasi, C., Manduchi, R.: Bilateral filtering for gray and color images. In: Proc. IEEE Int. Conf. Computer Vision, pp. 839-846 (1998) 